\title{
Is Tumor Size a Predictor of Survival in Stage IIA Cervical Cancer? A Comparison Between the Old and the New FIGO Staging Criteria
}

\author{
Yong Kuei Lim ${ }^{*}$, Hui Xian Chin ${ }^{1}$, Xiao Hui Lin ${ }^{1}$, Kwai Lam Yam${ }^{1}$, Yin Nin Chia ${ }^{1}$, Hoon Seng Khoo Tan ${ }^{2}$, Ming Chert \\ Richard Yeo ${ }^{2}$, Swee Peng Yap ${ }^{2}$, Lay Tin Soh ${ }^{2}$ and Eu Jin Chua ${ }^{2}$
}

${ }^{1}$ Department of Gynecological Oncology, KK Women's and Children's Hospital, 100 Bukit Timah Road, 229899, Singapore

${ }^{2}$ Division of Radiation Oncology, National Cancer Centre, 11 Hospital Drive, 169610, Singapore

\begin{abstract}
Objectives: In light of studies suggesting that tumor size is a prognostic factor for Stage IIA cervical cancers, FIGO revised the cervical cancer staging criteria, subdividing Stage IIA into Stages IIA1 and IIA2, based on tumor size $(<=4 \mathrm{~cm}$ and $>4 \mathrm{~cm}$ ). This study aims to determine if the new sub-staging has any impact on overall survival as well as elucidate any other prognostic factors and treatment patterns in this category of patients.

Materials and Methods: This is a case series of women diagnosed with Stage IIA cervical cancer in KK Women's \& Children's Hospital between 2001 and 2005. Statistical analysis was performed with SPSS version 19.

Results: A total of 66 patients were diagnosed with Stage IIA cervical cancer between 2001 and 2005 . Of the 50 patients which met the inclusion criteria, 35 (70\%) had stage IIA1 and 15 (30\%) had Stage IIA2 cervical cancer. The overall 5 year survival was not significantly different between Stage IIA1 and IIA2 $(79.6 \%$ vs $73.3 \%, p=0.9)$. Likewise, progression free survival was not significantly different between Stage IIA1 and IIA2 ( $76 \%$ vs $80 \%, \mathrm{p}=0.86)$. Patients who underwent radical hysterectomy performed better than those treated by primary radiotherapy ( $87 \%$ vs $69.6 \%, \mathrm{p}=0.043)$. Cox regression analysis showed that age, tumor grade, histology types, tumor size and lymph node status were not independent predictors of survival.

Conclusion: Our preliminary results did not find any difference in overall survival based on the new FIGO sub-staging of IIA1 and IIA2. Larger studies are needed to confirm this finding.
\end{abstract}

\section{Introduction}

Cervical cancer is the 10th most common female cancer in Singapore [1], with about 190 cases diagnosed every year. Approximately 130 cases of cervical cancer are diagnosed each year in our institution, KK Women's and Children's Hospital, of which $10 \%$ are Stage IIA. Cervical cancer is staged clinically in our institution, using the International Federation of Gynecology and Obstetrics (FIGO) staging criteria.

In light of studies suggesting that tumor size is a prognostic factor for Stage IIA cervical cancers, FIGO revised the cervical cancer staging criteria [2], subdividing stage IIA into stages IIA1 and IIA2, based on tumor size $(<=4 \mathrm{~cm}$ and $>4 \mathrm{~cm})$ in 2009 .

Subsequently, several studies have attempted to validate the changes in the FIGO staging criteria, but with varying results. This study aims to determine if the new sub-staging has any impact on overall survival, as well as to elucidate any other prognostic factors and treatment patterns in this category of patients at our institution.

\section{Materials and Methods}

This was a retrospective cohort study conducted in KK Women's and Children's Hospital, a large tertiary institution in Singapore. The sample consisted of all women who were diagnosed with FIGO Stage IIA cervical cancer from 1st January 2001 to 31st December 2005. The medical information required was retrieved from the KK Gynecological Cancer Centre Database as well as patient records.

The inclusion criteria included 1) tumor histology of squamous, adenocarcinoma or adenosquamous, 2) documented clinical tumor size, and 3) patients who received primary treatment in KK Hospital.
Primary treatment included either radical hysterectomy (RH) or radiotherapy (RT), with or without, concurrent chemotherapy.

At our centre, Stage IIA cervical cancer patients who have undergone primary $\mathrm{RH}$ are considered for adjuvant therapy depending on surgical pathological information. In the presence of positive lymph nodes, parametrial involvement and positive surgical margins, postoperative radiation with concurrent chemotherapy is given to reduce the risk of both local and distant failure [3].

In the group of patients considered to be of "intermediate" risk, as identified by Delgado et al. from Gynaecology Oncology Group (GOG), i.e. without the above the above adverse factors but with bulky disease, deep stromal invasion and lymphovascular space invasion, it is thought that adjuvant radiotherapy may be beneficial. The GOG score is calculated by multiplying the relative risks assigned for factors such as tumor size, capillary or lymphatic space involvement and depth of invasion [4]. Based on the score obtained, patients would be recommended either observation, small field radiotherapy or standard field radiotherapy [5].

"Corresponding Author: Dr. Yong Kuei Lim, Department of Gynecological Oncology, KK Women's and Children's Hospital, 100 Bukit Timah Road, 229899. Singapore; E-mail: timothy.lim.y.k@singhealth.com.sg

Citation: Lim YK, Chin HX, Lin XH, Yam KL, Chia YN, et al. (2017) Is Tumor Size a Predictor of Survival in Stage IIA Cervical Cancer? A Comparison Between the Old and the New FIGO Staging Criteria. Int J Gynecol Clin Pract 4: 128. doi: https://doi.org/10.15344/2394-4986/2017/128

Copyright: @ $2017 \mathrm{Lim}$ et al. This is an open-access article distributed under the terms of the Creative Commons Attribution License, which permits unrestricted use, distribution, and reproduction in any medium, provided the original author and source are credited. 
Citation: Lim YK, Chin HX, Lin XH, Yam KL, Chia YN, et al. (2017) Is Tumor Size a Predictor of Survival in Stage IIA Cervical Cancer? A Comparison Between the Old and the New FIGO Staging Criteria. Int J Gynecol Clin Pract 4: 128. doi: https://doi.org/10.15344/2394-4986/2017/128

Page 2 of 4

The patients were retrospectively assigned using the 2009 FIGO staging criteria to IIA1 and IIA2. The clinico-pathological factors analyzed include patient demographics, histology types, grade of tumor, tumor size, lymphovascular space invasion, lymph node status, type of primary treatment modality and adjuvant treatment. Characteristics between stage IIA1 and stage IIA2 were compared with the Pearson $\mathrm{x} 2$ test.

Overall survival and disease free interval were calculated using the Kaplan-Meier method. Cox regression hazard model was used to determine the prognostic factors. The statistical analysis was performed using SPSS version 19.

\section{Results}

A total of 66 patients were diagnosed with Stage IIA cervical cancer between 2001 and 2005. Of the 50 patients who met the inclusion criteria, $35(70 \%)$ had Stage IIA1 and $15(30 \%)$ had Stage IIA 2 cervical cancer. See Table 1 for patient demographics. None of the patients were lost to follow-up.

The mean age at diagnosis was 55.8 years and the patients who had Stage IIA2 were younger than those with Stage IIA1 though this was not statistically significant $(\mathrm{p}=0.12)$. The majority of the patients were Chinese (90\%) and the most common tumor histology was squamous cell carcinoma (80\%).

A total of 23 patients (46\%) underwent primary $\mathrm{RH}$, of which 16 were Stage IIA1 and 7 were Stage IIA2. A total of 27 patients (54\%) underwent primary RT, of which 19 are Stage IIA1 and 8 are Stage
IIA2. There was no significant difference between the two groups in terms of primary treatment modality (45.7\% Stage IIA1 vs $46.7 \%$ Stage IIA2 underwent primary $\mathrm{RH}, \mathrm{p}=0.50$ ).

Among the patients who underwent primary $\mathrm{RH}$, the lymph node metastases were found in $52 \%(12 / 23)$ of patients. However there was no statistically significant difference in lymph node metastases between Stage IIA1 and Stage IIA2 (50 \% vs 57\%, p=1.0). Almost all the patients (95.7\%) who had primary RH received adjuvant radiotherapy. This was not significantly different between Stage IIA1 and Stage IIA2 (93.8\% vs $100 \%, \mathrm{p}=1.0)$.

The 5-year overall survival (OS) was not significantly different between Stage IIA1 and IIA2 (79.6\% vs $73.3 \%, \mathrm{p}=0.9)$ (Figure 1). Likewise, the progression-free survival was not significantly different between Stage IIA1 and IIA2 ( $76 \%$ vs $80 \%, \mathrm{p}=0.86$ ) (Figure 2).

However, patients who underwent primary $\mathrm{RH}$ performed better compared to those treated by primary RT or concurrent chemoradiotherapy ( $87 \%$ vs $69.6 \%, \mathrm{p}=0.043)$.

Cox regression analysis showed that age, tumor grade, histology types, tumor size and lymph node status were not independent predictors of survival.

\section{Discussion}

In 2007, Horn et al. [6] showed that tumor size, defining bulky disease as tumors larger than $4 \mathrm{~cm}$, is of prognostic impact in FIGO stage II cervical carcinomas. This study showed that patients with tumors

\begin{tabular}{|c|c|c|c|c|c|}
\hline Variable & & $\begin{array}{l}\text { Stage IIA } \\
(\mathrm{n}=50)\end{array}$ & $\begin{array}{l}\text { Stage IIA1 } \\
(\mathrm{n}=35)\end{array}$ & $\begin{array}{l}\text { Stage IIA2 } \\
(\mathrm{n}=15)\end{array}$ & $\mathrm{P}$ \\
\hline Mean age in years (range) & & $55.9(35-84)$ & $57.5(35-84)$ & $52.1(37-67)$ & 0.12 \\
\hline \multirow[t]{2}{*}{ Race } & Chinese & $45(90 \%)$ & $32(91.4 \%)$ & $13(86.7 \%)$ & 0.63 \\
\hline & Malay & $5(10 \%)$ & $3(8.6 \%)$ & $2(13.3 \%)$ & \\
\hline \multirow[t]{3}{*}{ Histology } & Squamous & $40(80 \%)$ & $29(82.9 \%)$ & $11(73.3)$ & 0.149 \\
\hline & Adenocarcinoma & $6(12 \%)$ & $5(8.6 \%)$ & $1(6.7 \%)$ & \\
\hline & Adenosquamous & $4(8 \%)$ & $1(2.9 \%)$ & $3(20 \%)$ & \\
\hline \multirow[t]{4}{*}{ Tumour grade } & Grade 1 & $3(6 \%)$ & $1(2.8 \%)$ & $2(13.3 \%)$ & 0.437 \\
\hline & Grade 2 & $21(42 \%)$ & $14(40 \%)$ & $7(46.7 \%)$ & \\
\hline & Grade 3 & $25(50 \%)$ & $19(54.3 \%)$ & $6(40 \%)$ & \\
\hline & Ungrade & $1(2 \%)$ & $1(2.9 \%)$ & $0(0 \%)$ & \\
\hline \multirow[t]{4}{*}{ Tumour Size } & $<2 \mathrm{~cm}$ & $8(16 \%)$ & $8(22.9 \%)$ & $0(0 \%)$ & $<0.001$ \\
\hline & $2-4 \mathrm{~cm}$ & $28(56 \%)$ & $27(77.1 \%)$ & $1(6.7 \%)$ & \\
\hline & $4-6 \mathrm{~cm}$ & $11(22 \%)$ & $0(0 \%)$ & $11(73.3 \%)$ & \\
\hline & $>6 \mathrm{~cm}$ & $3(6 \%)$ & $0(0 \%)$ & $3(20 \%)$ & \\
\hline \multirow[t]{2}{*}{ Treatment } & Radical surgery & $23(46 \%)$ & $16(45.7 \%)$ & $7(46.7 \%)$ & 0.50 \\
\hline & Primary RT & $27(54 \%)$ & $19(54.3 \%)$ & $8(53.3 \%)$ & \\
\hline \multirow[t]{2}{*}{ Adjuvant RT } & Yes & $22(95.7 \%)$ & $15(93.8 \%)$ & $7(100 \%)$ & 1.0 \\
\hline & No & $1(4.3 \%)$ & $1(6.3 \%)$ & 0 & \\
\hline \multirow[t]{2}{*}{ Surgery + Adjuvant treatment } & RT alone & $5(22.7 \%)$ & $3(20 \%)$ & $2(28.6 \%)$ & 0.655 \\
\hline & ChemoRT & $17(77.3)$ & $12(80 \%)$ & $5(71.4 \%)$ & \\
\hline \multirow[t]{2}{*}{ Primary RT } & RT alone & $16(59.3 \%)$ & $15(78.9 \%)$ & $1(12.5 \%)$ & 0.001 \\
\hline & ChemoRT & $11(40.7 \%)$ & $4(21.1 \%)$ & $7(87.5 \%)$ & \\
\hline
\end{tabular}

Table 1: Demographic and clinicopathological variables by stage. 
Citation: Lim YK, Chin HX, Lin XH, Yam KL, Chia YN, et al. (2017) Is Tumor Size a Predictor of Survival in Stage IIA Cervical Cancer? A Comparison Between the Old and the New FIGO Staging Criteria. Int J Gynecol Clin Pract 4: 128. doi: https://doi.org/10.15344/2394-4986/2017/128

Page 3 of 4

$>4 \mathrm{~cm}$ showed an increase of recurrent disease compared to tumors $<$ or $=4 \mathrm{~cm}(40.2 \%$ vs. $28.0 \%$; $\mathrm{p}=0.045)$. The 5 -year OS rate was also significantly lower $(67.7 \%$ vs. $49.5 \%$; $\mathrm{p}=0.0015)$ in patients with tumors $>4 \mathrm{~cm}$. In multivariate analysis, tumor stage, pelvic lymph node involvement and maximal tumor size were independent prognostic factors. All 245 patients with Stage IIA or IIB in this study underwent upfront surgery, but this is not routinely performed in many centres around the world where radiotherapy is the standard treatment for most cases of Stage II cancers. Nevertheless, the FIGO classification of Stage IIA was revised to Stage IIA1 and IIA2 in 2009.
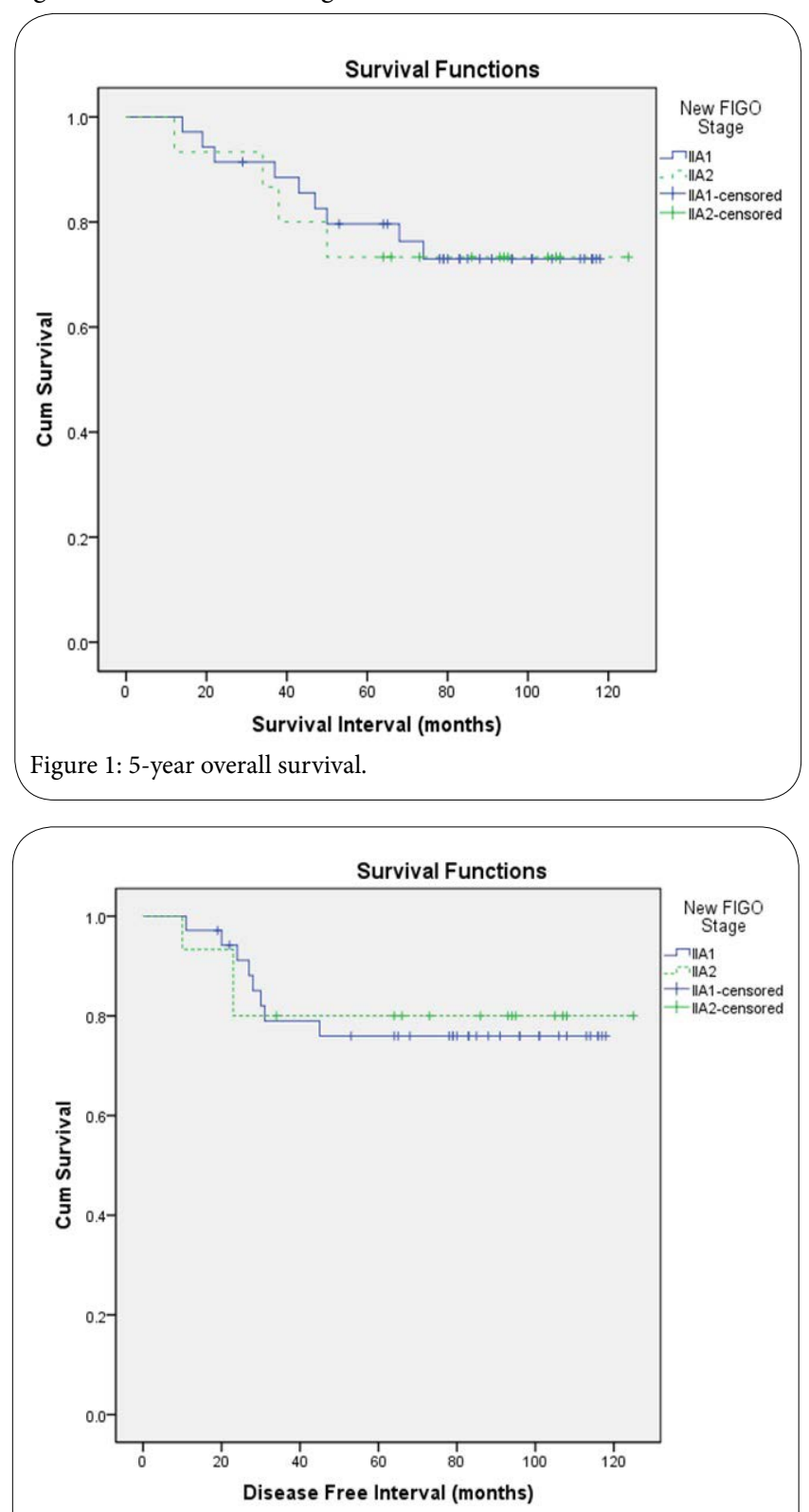

Figure 2: Progression-free survival.

Our study did not find any difference in progression-free or 5-year OS based on the 2009 FIGO sub-staging of IIA1 and IIA2. Similarly, in a study of 560 patients with Stage IIA cervical cancer (data extracted from SEER database from 1988 to 2005; 271 with Stage IIA1 and 289 with Stage IIA2 disease) by Garg et al7, no significant difference in survival was found. The 5-year OS was $65.8 \%$ in Stage IIA1 vs $59.5 \%$ in Stage IIA2 $(\mathrm{p}=0.2)$. Likewise, in a study by Lai et al 8 , which included 51 patients with Stage IIA cervical cancer (36 with Stage IIA1 and 15 with Stage IIA2 disease), there was no significant difference in survival between the two groups $(86.3 \%$ vs $51.9 \%, \mathrm{p}=0.218)$. In these studies, primary treatment included either RH or RT.

In another recent study, Hongladaromp et al. [9] looked into 133 patients with Stage IIA cervical cancer, 101 (75.9\%) Stage IIA1, and 32 (24.1\%) Stage IIA2. All of these patients were treated with $\mathrm{RH}$ and pelvic lymphadenectomy. There were comparable rates of 5-year disease-free survival ( $84.6 \%$ vs $88.7 \%, \mathrm{p}=0.67)$ and 5 -year OS $(83.4 \%$ vs $90.0 \%, \mathrm{P}=0.49$ ). The authors hence concluded that the revised 2009 FIGO staging system did not demonstrate significant survival differences between Stage IIA1 and Stage IIA2 cervical cancer.

On the other hand, Wagner et al. [10] demonstrated in a larger study of 857 patients with Stage IIA cervical cancer (data extracted from SEER database from 1988 to 2008), that patients with Stage IIA1 disease had improved cause-specific survival (CSS) compared to patients with Stage IIA2, with 5-year CSS's of $84 \%$ and $69 \%$ respectively $(\mathrm{p}<0.0001)$. Mean survival times were 146 months for Stage IIA1 compared to 73 months for Stage IIA2 ( $<<0.0001)$. This supports the changes made to cervical cancer staging 2009. It is likely that Wagner et al's results differed from the other studies due to several reasons, such as the larger number of patients included, as well as the use of CSS, instead of OS, as the primary endpoint of analysis, hence reducing the risk of other confounding variables.

Garg et al. concluded that age, tumor size and lymph node status were independent predictors of survival and Wagner et al concurred that age was significantly and independently prognostic. On the other hand, this was not shown by Lai et al, nor in our study. This is likely due to the smaller numbers in the latter studies. Moreover, at our centre, clinical staging is routinely performed via examination under anesthesia. Tumor size is measured clinically and not dependent on radiology, which is more reflective of what is recommended in the FIGO clinical staging criteria.

In terms of treatment modalities, Stage IIA cervical cancer is treated with either primary $\mathrm{RH}$ or RT with or without concurrent chemotherapy. Primary surgical treatment allows preservation of sexual function and ovarian conservation, avoids the late side effects of radiotherapy, and is hence preferred in younger patients. The decision on primary treatment is dependent on the policy of the institution, gynecological oncologist, radiation oncologist, age and general health of the patient. Our study shows that only age at diagnosis was found to be a significant predictor of primary surgical treatment $(\mathrm{p}=0.009)$ and RHs were more commonly performed in younger patients below the age of 60 .

Patients who underwent primary $\mathrm{RH}$ in our study were found to perform significantly better compared to those treated by primary RT ( 5 -year OS $87 \%$ vs $69.6 \%, p=0.043$ ). This was likely due to the fact that $95.7 \%$ of patients who underwent primary $\mathrm{RH}$ in our study received adjuvant treatment with RT or concurrent chemo-radiotherapy $(p<0.05)$, based on the postoperative GOG score. It is important to be mindful that patients who undergo primary $\mathrm{RH}$, followed by adjuvant RT with or without chemotherapy, are subjected to complications of both treatment modalities hence increased morbidity11. Further studies are needed to aid in streamlining the selection of patients for each treatment arm, so as to decrease morbidity and improve quality of life after treatment.

On the other hand, the study by Garg et al showed that there was no significant difference in survival between patients treated by surgery 
Citation: Lim YK, Chin HX, Lin XH, Yam KL, Chia YN, et al. (2017) Is Tumor Size a Predictor of Survival in Stage IIA Cervical Cancer? A Comparison Between the Old and the New FIGO Staging Criteria. Int J Gynecol Clin Pract 4: 128. doi: https://doi.org/10.15344/2394-4986/2017/128

Page 4 of 4

and radiotherapy ( $p>0.05)$, with $67.3 \%$ of patients being administered adjuvant radiotherapy. However, the incidence of the use of concurrent chemo-radiation as adjuvant therapy was not reported. Similarly, Lai et al. did not show statistical significance in the survival between the different treatment modalities. This may be due to the limited number of patients with Stage IIA2 disease in this study (36 Stage IIA1 vs 15 Stage IIA2), a higher proportion of $71 \%$ of patients undergoing primary $\mathrm{RH}$, and also the lack of information on types of $\mathrm{RH}$ and details of chemotherapy. The study by Wagner et al was unable to evaluate the benefit of different treatment modalities, as complete details on surgery, radiation and chemotherapy were not available.

\section{Conclusions}

In conclusion, our study did not find any difference in overall survival based on the new FIGO sub-staging of IIA1 and IIA2 in our local population. However, it is important to bear in mind that this study is small and retrospective, hence it may not have sufficient power to show any difference. Larger prospective studies are needed to confirm our findings.

\section{Competing Interests}

The authors declare that they have no competing interests.

\section{References}

1. National Registry of Diseases Office Singapore Cancer Registry Annual Registry Report: Trends in Cancer Incidence in Singapore 2009 - 2013.

2. Pecorelli S, Zigliani L, Odicino F (2009) Revised FIGO staging for carcinoma of the cervix. Int J Gynaecol Obstet 105: 107-108.

3. Peters WA, Liu PY, Barrett RJ , Stock RJ, Monk BJ, Berek JS, et al. (2000) Concurrent chemotherapy and pelvic radiation therapy alone as adjuvant therapy after radical surgery in high risk early stage cancer of the cervix. $J$ Clin Oncol $18:$ 1606-1613.

4. Delgado G, Bundy B, Zaino R, Sevin BU, Creasman WT, et al. (1990) Prospective surgical-pathological study of disease free-interval in patients with stage lb squamous cell carcinoma of the cervix: a Gynecologic Oncology Group study. Gynecol Oncol 38: 352-379.

5. Yeo RM, Chia YN, Namuduri RP, Yap SP, Soong YL, et al.(2011) Tailoring adjuvant radiotherapy for stage IB-IIA node negative cervical carcinoma after radical hysterectomy and pelvic lymph node dissection using the GOG score. Gynecol Oncol 123: 225-229.

6. Horn LC, Fischer U, Raptis G, Bilek K, Hentschel B, et al. (2007) Tumor size is of prognostic value in surgically treated FIGO stage II cervical cancer. Gynecol Oncol 107: 310-315.

7. Garg G, Shah JP, Toy EP, Christensen C, Deppe G, et al. (2011) Stage IIA1 versus stage IIA2 cervical cancer: does the new staging criteria predict survival? Int J Gynecol Cancer 21: 711-716.

8. Lai JC, Chou YJ, Huang N, Tsai JJ, Huang SM, et al. (2013) Survival analysis of Stage IIA1 and IIA2 cervical cancer patients. Taiwan J Obstet Gynecol 52: 33-38.

9. Hongladaromp W, Tantipalakorn C, Charoenkwan K, et al. (2014) Locoregional spread and survival of stage IIA1 versus stage IIA2 cervical cancer. Asian Pac J Cancer Prev 15: 887-890.

10. Wagner AE, Pappas L, Ghia AJ, Gaffney DK (2013) Impact of tumor size on survival in cancer of the cervix and validation of stage IIA1 and IIA2 subdivisions. Gynecol Oncol $129: 517-521$.

11. Landoni F, Maneo A, Colombo A, Placa F, Milani R, et al. (1997) Randomised study of radical surgery versus radiotherapy for stage lb-lla cervical cancer. Lancet 350: 535-540.

This article was originally published in a special issue: Gynecology \& Surgical Oncology

Handled by Editor(s):

Dr. Maria Teresa Gomez Garcia

Department of Medical Sciences University of Castile-La Mancha Spain 